\title{
Gamma-ray astronomy and cosmic-ray physics with ARGO-YBJ
}

\author{
P. Camarri*i \\ University of Roma "Tor Vergata" and INFN Roma Tor Vergata \\ E-mail: paolo.camarridroma2.infn.it
}

The ARGO-YBJ detector, located $4300 \mathrm{~m}$ a.s.l. on the Tibet plateau, is a ground-based, fullcoverage array of Resistive Plate Chambers (RPCs) covering a surface of $78 \times 74 \mathrm{~m}^{2}$, surrounded by a guard ring of RPCs enclosing a total surface of about $11000 \mathrm{~m}^{2}$. ARGO-YBJ was designed to detect extensive air showers generated by cosmic rays and gamma rays with primary energy greater than few hundred $\mathrm{GeV}$, in order to study the region of the cosmic-ray spectrum out of the reach of both satellite-based experiments and traditional ground-based arrays. The experiment has been running with its complete layout since November 2007, collecting over $2.5 \times 10^{11}$ events. The main results obtained by ARGO-YBJ will be presented here, and specifically: the monitoring of astronomical gamma-ray sources, such as the Crab nebula and the MRK 421 AGN, the moon shadow, the medium-scale anisotropy map, the proton-proton inelastic cross section at center-ofmass energy between 70 and $500 \mathrm{GeV}$ where no accelerator data are available.

The 2011 Europhysics Conference on High Energy Physics, EPS-HEP 2011,

July 21-27, 2011

Grenoble, Rhône-Alpes, France

* Speaker.

† on behalf of the ARGO-YBJ Collaboration. 


\section{ARGO-YBJ and its goals}

The ARGO-YBJ experiment [ $[$ ] is a collaboration between Chinese and Italian research groups. It is located in Tibet, China, at an altitude of $4300 \mathrm{~m}$ a.s.l. in order to reconstruct air showers generated by cosmic rays with energy down to a few hundred GeV. ARGO-YBJ was designed to investigate a large number of topics in astrophysics and cosmic-ray physics: $\gamma$-ray astronomy (search for point-like sources above few hundreds of $\mathrm{GeV}$ ), search for VHE tails of $\gamma$-ray bursts above $\sim 1$ $\mathrm{GeV}$, cosmic-ray physics, Sun and heliosphere physics.

The ARGO-YBJ detector is based on a full-coverage single layer of Resistive Plate Chambers (RPCs) covering a surface of $74 \times 78 \mathrm{~m}^{2}$. A group of 12 neighboring RPCs is called a "cluster", and the full-coverage part of the detector includes 130 clusters. Around this, 23 additional clusters are placed in order to obtain a better reconstruction of the shower front, which extends the experiment area to $110 \times 100 \mathrm{~m}^{2}$. The space-time unit of the ARGO-YBJ detector is a "pad", namely a group of 8 neighboring RPC read-out strips. The single-hit time resolution is about $1.8 \mathrm{~ns}$ []]. Air showers are triggered by requiring a number of hits greater than 20 within a $150 \mathrm{~ns}$ time window, giving an average trigger rate of about $3.5 \mathrm{kHz}$ with a dead time of $4 \%$ and an average duty cycle greater than $86 \%$.

ARGO-YBJ has been running almost uninterruptedly with its complete layout since October 2007 with a duty cycle of $90 \%$ and an average trigger rate of $3.6 \mathrm{kHz}$. A selection of the main physics results obtained so far is presented here.

\section{2. $\gamma$-ray astronomy with ARGO-YBJ}

The analysis of the ARGO-YBJ data in $\gamma$-ray astronomy in the first three years of running was mainly focused on the study of known sources, on the study of AGN flares, and on the limits on VHE fluence from $\gamma$-ray bursts. The background was evaluated by using two independent, widely established procedures: the "time-swapping" method and the "equi-zenith" method, which give equivalent results.

The $\gamma$-ray emission from the Crab Nebula was detected with a significance of about 14.5 standard deviations in 800 days. Figure $\square$ shows the experimental points measured by ARGO-YBJ on the Crab Nebula energy spectrum, in perfect agreement with the known experimental results obtained by the HESS, MAGIC, Whipple and Veritas experiments.

A few remarkable flares from the AGN MRK421 were observed in the X-ray range by the ASM X-ray telescope in 2006, 2008 and 2010, and ARGO could observe TeV gamma emission at the same time for such flares as shown in fig $\square$. Concerning the two flares observed on June 4-6 and June 11-13, 2008 [3] ], ARGO-YBJ investigated the gamma emission from the same source on those days [四]. The peak significance for the flare of June 11-13, 2008 was 4.2 standard deviations. The ARGO-YBJ data fully satisfy the relation between the spectral index and the flux resulting from the Whipple measurements of a similar flare of MRK421 [5], suggesting that this relation is an intrinsic property of the source. One more remarkable flare from MRK421 occurred on February 2010. ARGO-YBJ observed it between February 16th and February 18th at a 6 s.d. significance level. The measured flux exceeded $3 \mathrm{Crab}$ units for the duration of the observation. The importance 


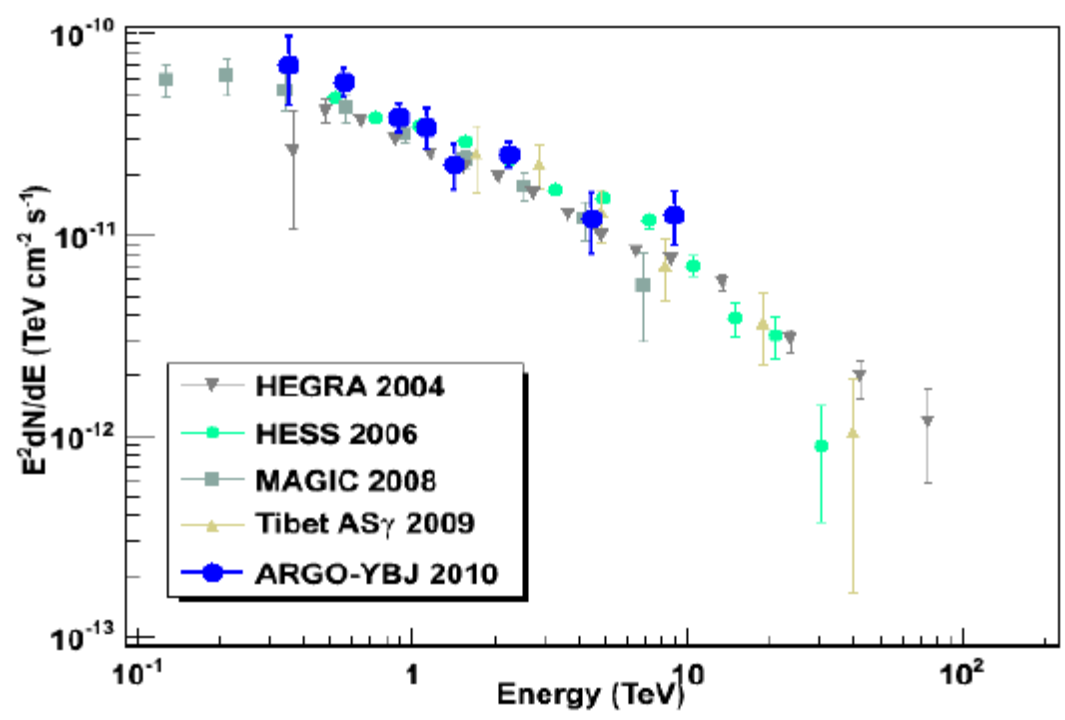

Figure 1: Gamma-ray spectrum from the Crab Nebula measured by ARGO-YBJ in 2010, compared with the results obtained by HEGRA, HESS, MAGIC and Tibet AS- $\gamma$.

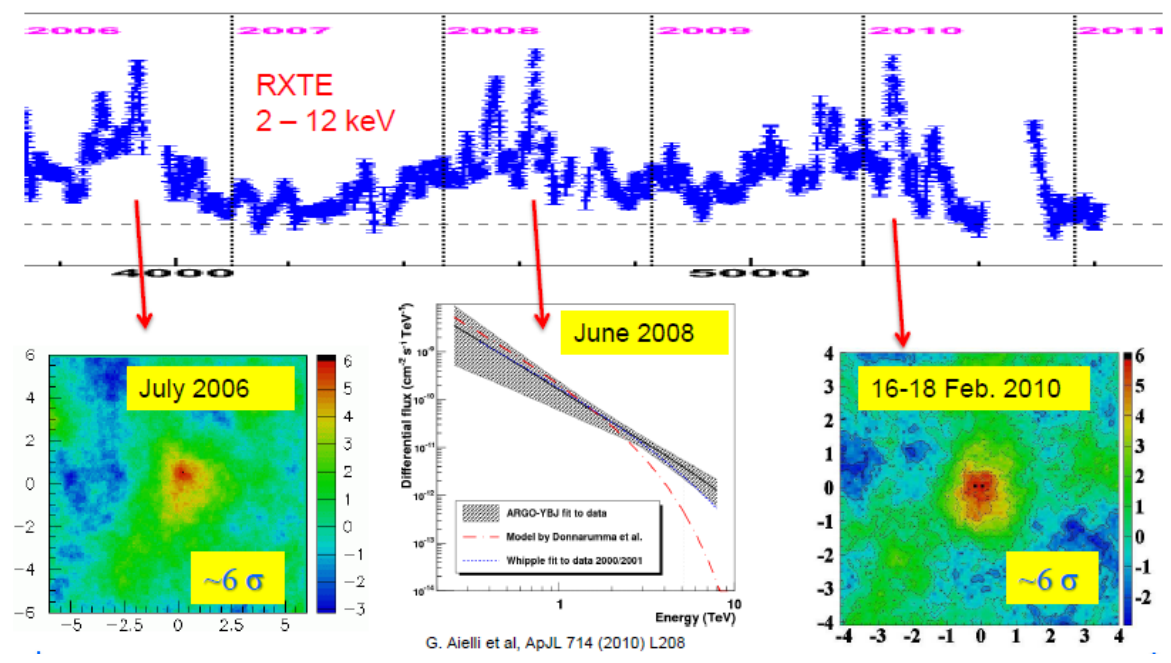

Figure 2: Gamma-ray emission from Mrk421 measured by ARGO-YBJ at the times when X-ray flares were detected by the ASM X-ray telescope in 2006, 2008 and 2010. On 2008, June 11-13 the gamma-emission spectrum could be measured (solid line). The shaded band represents the one-standard-deviation error. The dot-dashed line shows the flux according to the model by Donnarumma et al. [3] for the second flare (June 12-13). The dotted line shows the spectrum measured by Whipple [ [ [] during a previous flare of similar intensity. 


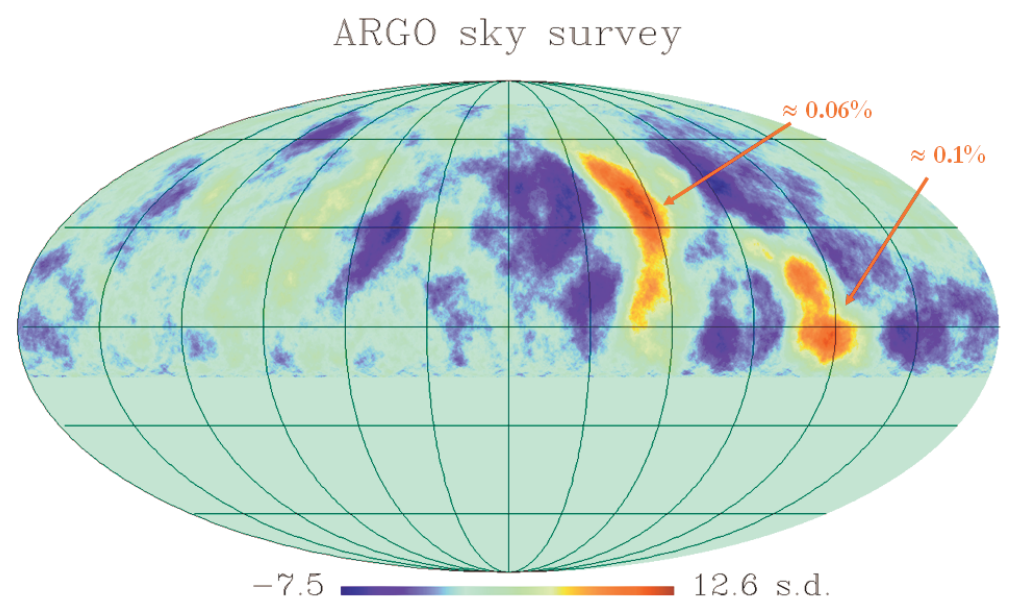

Figure 3: ARGO-YBJ all-sky significance map for events collected between day 311, 2007 and day 220, 2009 with more than 40 hit pads. A smoothing window radius of $5^{\circ}$ was used.

of this measurement by ARGO-YBJ lies in the fact that for the first time a ground-based experiment could detect a flare with a 5 s.d. significance level on a daily basis.

At present ARGO-YBJ is performing a thorough full-sky search for more $\gamma$-ray sources.

\section{Cosmic-ray physics with ARGO-YBJ}

The studies in cosmic-ray physics with ARGO-YBJ were focused so far on the following items: search for anisotropy at $\mathrm{TeV}$ energies, study of the moon shadow and the corresponding limit on the $\bar{p} / p$ flux ratio, proton-air interaction cross section.

Concerning the medium-scale anisotropy, the ARGO-YBJ significance map of the whole visible sky with cosmic rays is shown in Fig. [1] [目]. For this study, the data collected between 2007 and 2010 were used. A number of hit pads greater than 40 and a zenith angle $\theta<40^{\circ}$ were the requests for this analysis (corresponding to a median energy of about $2 \mathrm{TeV}$ ), and the map was obtained by using a smoothing window radius of $5^{\circ}$. Two major excess regions are clearly visible, with relative excesses of about $0.06 \%$ for the leftmost region and $0.1 \%$ for the rightmost region. These regions correspond respectively to region $\mathrm{B}$ and region $\mathrm{A}$ of the all-sky significance map obtained by the MILAGRO experiment [ []]. Therefore, ARGO-YBJ confirms the result obtained by MILAGRO for the medium-scale anisotropy. A possible explanation for this puzzling experimental evidence will be the subject of future studies.

An important subject of study in cosmic-ray physics is the shadowing effect exerted by the Moon on primary cosmic rays. Ground-based experiments can measure three crucial quantities related to this effect: the size, the position and the westward displacement of the deficit. From these measurements, information on the angular resolution, the pointing accuracy and the energy calibration of the detector respectively can be extracted. Concerning the last of these three issues, the basic procedure is based on the angular bending $\Delta \theta$ of a primary cosmic ray with energy $E$ 
and atomic number $Z$ in the geomagnetic field between the Moon and the Earth: $\Delta \theta \simeq 1.57^{\circ}$. $Z / E(\mathrm{TeV})$. The results coming from the data collected until the end of 2010 were described in detail in [[8].

Another significant result is the measurement of the proton-air interaction cross section at energies ranging from a few $\mathrm{TeV}$ to $100 \mathrm{TeV}$. The expected flux of atmospheric showers versus the zenith angle $\theta$ is

$$
I(\theta)=I(0) \cdot e^{-\frac{h_{0}}{\Lambda}(\sec \theta-1)}
$$

where $h_{0}$ is the atmospheric depth of the experimental setup $\left(606.7 \mathrm{~g} / \mathrm{cm}^{2}\right.$ for ARGO-YBJ) and $\Lambda$ is the proton absorption length in air. It differs from the proton interaction length $\lambda_{\text {int }}$ mainly because of collision inelasticity, shower fluctuations and detector resolution. It can be shown that $\Lambda=k \lambda_{\text {int }}$, where $k$ must be determined by simulations. The proton-air interaction cross section is given by

$$
\sigma_{p-A i r}=2.4 \cdot 10^{4} / \lambda_{\text {int }}\left(\mathrm{g} / \mathrm{cm}^{2}\right)
$$

and the corresponding proton-proton cross section can be obtained from this, at center-of-mass energy between 70 and $500 \mathrm{GeV}$ where no data from accelerator experiments are available. The result of this study was described in detail in [Q].

\section{References}

[1] G. Aielli et al.; NIM A 562 (2006) 92-96.

[2] G. Aielli et al.; NIM A 608 (2006) 246-250.

[3] I. Donnarumma et al.; ApJ 691 (2009) L13-L19.

[4] G. Aielli et al. (the ARGO-YBJ collaboration); ApJ Letters 714 (2010) L208-L212.

[5] F. Krennrich et al. (the WHIPPLE collaboration); ApJ 575 (2002) L9-L13.

[6] G. Di Sciascio on behalf of the ARGO-YBJ collaboration; arXiv: 1010.4401.

[7] A. A. Abdo et al. (the MILAGRO collaboration); Phys. Rev. Lett. 101 (2008) 221101.

[8] B. Bartoli et al. (the ARGO-YBJ collaboration); Phys. Rev. D 84 (2011) 022003.

[9] G. Aielli et al. (the ARGO-YBJ collaboration); Phys. Rev. D 80 (2009) 092004. 